\title{
Three-Dimensional Reconstruction and Surgical Navigation in Padiatric Epilepsy Surgery
}

\author{
Alexandra Chabrerie ${ }^{1,3}$, Fatma Ozlen ${ }^{1,3}$, Shin Nakajima ${ }^{1,3}$, \\ Michael Leventon ${ }^{2}$, Hideki Atsumi ${ }^{1}$, Eric Grimson ${ }^{2}$, Erwin Keeve ${ }^{1}$, \\ Sandra Helmers ${ }^{4}$, James Riviello Jr. ${ }^{4}$, Gregory Holmes ${ }^{4}$, Frank Duffy ${ }^{4}$, \\ Ferenc Jolesz ${ }^{1}$, Ron Kikinis ${ }^{1}$, and Peter Black ${ }^{3}$ \\ 1. Surgical Planning Laboratory, Brigham and Women's Hospital, \\ Harvard Medical School, Boston, MA \\ 2 Artificial Intelligence Laboratory, \\ Massachusetts Institute of Technology, Cambridge, MA \\ 3 Division of Neurosurgery, Brigham and Women's Hospital, Children's Hospital, \\ Harvard Medical School, Boston, MA \\ 4 Department of Neurology, Children's Hospital, \\ Harvard Medical School, Boston, MA
}

\begin{abstract}
We have used MRI-based three-dimensional (3D) reconstruction and a real-time, frameless, stereotactic navigation device to facilitate the removal of seizure foci in children suffering from intractable epilepsy. Using this system, the location of subdural grid and strip electrodes is recorded on the $3 \mathrm{D}$ model to facilitate focus localization and resection. Ten operations were performed - two girls and eight boys ranging in age from 3-17 - during which 3D reconstruction and surgical instrument tracking navigation was used. In all cases, the patients tolerated the procedure well and showed no post-operative neurological deficits.
\end{abstract}

\section{Introduction}

Although most children suffering from epilepsy have a good prognosis for remission, a small percentage of cases are resistant to conventional Anti-Epileptic Drugs. For these patients, surgical intervention for seizure focus removal to stop the seizures and to prevent further brain injury provides great hope [1]. Subdural grid and strip electrodes in chronic monitoring of the Electroencephalogram (EEG) have become more widely used as a means of accurately obtaining the necessary localization of the focus with a minimum morbidity [2]. Implanting subdural electrodes directly on the brain surface allows the epilepsy investigator to record a series of ictal and interictal recordings, with ample time for a complete and precise mapping [3]. In addition, cortical stimulation for localizing an epileptic focus also determines its relation to eloquent areas and surrounding cortical function [4].

Magnetic Resonance Imaging (MRI) is a highly reliable neuroimaging procedure in patients with partial or localized epilepsy [4]. For several years, we have been making computerized, 3D reconstructions of pre-operative MRI scan 




Fig. 1. The interface displayed on the computer monitor during surgery. The position of the LED probe is simultaneously displayed on the 3D model (white arrow). The position on the corresponding MRI splices is displayed in the axial, sagittal and coronal planes. The subdural electrodes are shown as black spheres.

data for surgical planning [5] and intraoperative navigation. We have recently developed a novel neurosurgical navigator in which medical image registration and instrument tracking automatically establishes correspondence between the medical images from MRI scans, the three-dimensional model and the actual patient environment [6]. Using this intraoperative guidance, medical instruments acting on the patient are localized in the 3D coordinate frame of the MRI imagery. Consequently, the surgeon is allowed to view a patient and at the same time display, in exact alignment, that view and all other surrounding internal structures on both the reconstructed model and three views on the original MRI scans. Most importantly, any point may be recorded and displayed on the $3 \mathrm{D}$ model as an arrow or simple dot (see Figure 1).

We have combined functional diagnostic tools including EEG results and subdural strip and grid electrodes results, with real-time, intra-operative imaging and $3 \mathrm{D}$ reconstruction to increase the precision of localization and subsequent resection of seizure foci. The 3D models constructed from pre-operative MRI scans allow pre-operative surgical planning as well as intraoperative navigation. The latter is used as guidance for the surgeon to the correct location of the abnormality and to establish its boundaries for safe resection without affecting neighboring areas and decreasing the invasion of eloquent cortex. Most impor- 
tantly, it allows the recording of the subdural grid and strip electrode positions on the $3 \mathrm{D}$ model and as a result, provides an accurate, $3 \mathrm{D}$ visualization. A precise functional and structural map of the patient's brain is thus created and used for pre-operative planning and intraoperative navigation, ultimately increasing the accuracy localization of the focus and decreasing the likelihood of post-operative neurological deficits.

\section{Methods}

Patients: 3D reconstructions using MR images were created and used for surgical planning in 10 pediatric patients (ages 3 - 18 yrs; 2 girls and 8 boys), all suffering from intractable seizures.

Subdural grid and strip placement: Criteria for surgical intervention and investigation using cortical surface electrodes included intractable seizures and a unilateral focus believed to be cortical, temporal or extra-temporal. The grid and strip electrodes most often used initially are either a $4 \times 8$ or $8 \times 8$ electrode contact grid array (AdTech Medical Instruments; Racine, Wisc., USA) and were modified depending on the result of the pre-operative assessment or operative limitations. Surface recordings of each electrode on the grid are obtained in the operating room to ensure good contact with the brain surface prior to closure.

MR data acquisition and transfer: The MR data were obtained by a 1.5 Tesla MR unit (Signa; GE Medical Systems, Milwaukee, WI). A series of 124 images of post-contrast, 3D SPGR (SPoiled Gradient Recalled acquisition in the steadystate, $1.5 \mathrm{~mm}$ thickness, $256 \times 256$ matrix of $220-240 \mathrm{~mm}$ FOV) were obtained. An $\mathrm{MR}$ angiogram in either the axial or the sagittal plane for $3 \mathrm{D}$ reconstruction of the vasculature was used. The velocity encoding (VENC) was chosen according to the specific pathology. For cortical/subcortical tumors, we chose $20 \mathrm{~cm} / \mathrm{sec}$ VENC to visualize small cortical vessels. For arterial information, we used 60 $\mathrm{cm} / \mathrm{sec}$. The data were digitally transferred to the workstation (SPARC 20; Sun Microsystems, Inc., Mountain View, CA) via a computer network.

Image processing: Each image was pre-processed to reduce noise using anisotropic diffusion filtering [7]. A segmentation based on signal intensities and voxel connectivity $[8,9]$ was then performed using one of the MR series (SPGR and MR angiogram). Imperfections in the 3D objects were corrected by manual editing. From these images, 3D models of the skin, brain, vessels, focal lesion and ventricles were reconstructed using the marching cubes algorithm and a surface rendering method [8-10]. These objects were then integrated and displayed on a computer workstation (Ultra-1; Sun Microsystems, Inc.) with a graphic accelerator (Creator 3D; Sun Microsystems Inc.) and 3D display software (LAVA; Sun Microsystems, Inc.). Each object thus may be rendered partially or completely transparent and oriented according to the viewer's choice. T2-weighted images and MR angiogram images can be registered to the base series (SPGR) using a 

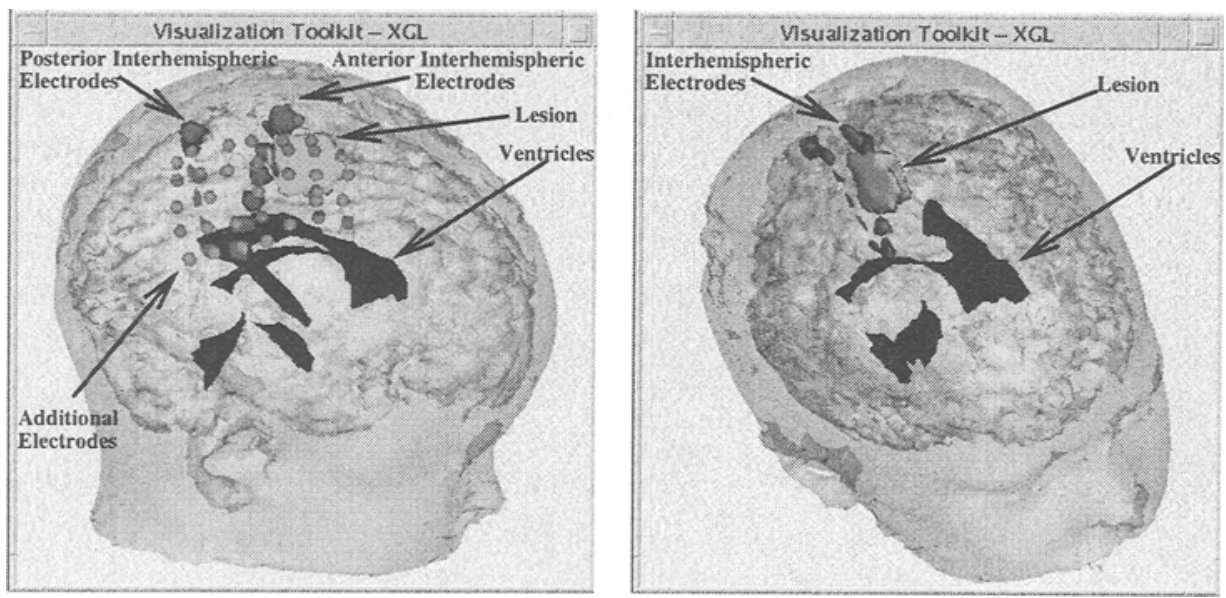

Fig. 2. 3D reconstruction showing the position of the subdural electrodes. 96 electrodes were applied directly to the cortical surface, including a posterior and an anterior interhemispheric strip. The parietal and frontal electrodes were localized using the trackable probe. The interhemispheric electrodes were segmented from an internal scan.

multi-modal volume registration method by maximization of mutual information [36]. Each object may be individually colored to facilitate visualization.

Surgical navigation: For intra-operative navigation, a real-time, Light Emitting Diode-based (LED), frameless, stereotactic device developed in the laboratory was used. Prior to surgery, a dynamic reference frame carrying three LED's (Image Guided Technologies, Inc.) is fixed next to the patient's head. A series of points from the patient's skin are recorded using an LED probe (Image Guided Technologies, Inc.). The information is tracked using an optical digitizer (Flashpoint 5000; Image Guided Technologies, Inc.). The points recorded in real space are then matched to the $3 \mathrm{D}$ model in two stages. An initial alignment is obtained by recording the real-space location of three points, then manually matching those points on the MRI model. This initial registration is refined by finding the optimal transformation that aligns all of the points onto the skin surface of the model $[6,14]$. After registration the surgeon points to a known area on the patient using the LED probe, to confirm correspondence between the patient and the 3D model and MRI slices. During surgery, the surgeon uses a sterile LED probe to select any point on or inside the patient's brain. Any point may be recorded and displayed on the $3 \mathrm{D}$ model using an arrow or simple dot (see Figure 2).

\section{Results and Illustrative Cases}

Ten patients were pre-operatively evaluated in the Surgical Planning Laboratory at Brigham and Women's Hospital, Boston, MA. Their ages ranged from 


\begin{tabular}{||l|l|l|l|l||}
\hline \hline Case & Diagnosis & Sex & Age & Outcome \\
\hline \hline 1 & Epilepsy & M & 7 & No new seizures \\
\hline 2 & Left frontal focus & M & 10 & No new seizures \\
\hline 3 & Left frontoparietal focus & M & 3 & No new seizures \\
\hline 4 & Hypothalamic Hamartoma & M & 18 & No new seizures \\
\hline 5 & Right parietal focus & M & 12 & No new seizures \\
\hline 6 & Temporal epilepsy & M & 15 & No new seizures \\
\hline 7 & Left temporal lobectomy & M & 8 & No new seizures \\
\hline 8 & Left temporal lobectomy & $\mathrm{F}$ & 5 & Severe seizures remaining \\
\hline 9 & Tuberous sclerosis & M & 10 & $\begin{array}{l}\text { Seizure-free for approx two months post- } \\
\text { operatively }\end{array}$ \\
\hline 10 & Tuberous sclerosis & F & 3 & $\begin{array}{l}\text { Seizures remaining but different in pattern } \\
\text { than during pre-operative evaluation }\end{array}$ \\
\hline \hline
\end{tabular}

Table 1. Summary of cases.

3 to 18 , including two females and eight males. The females included one left temporal lobectomy and one right frontal tuber removal. The males included one left subcortical tuber removal and callosotomy, one left temporal lobectomy and hippocampectomy, one right parietal lobe mass removal, one left frontoparietal focus removal, one temporal epilepsy, one hypothalamic hamartoma with gelastic seizures, one right frontal interhemispheric focus removal (Table 1). The pre-operative and post-operative diagnosis was identical in all patients. Seven remained seizure free post-operatively. One girl suffering from tuberous sclerosis still experienced seizures but different in pattern from those recorded preoperatively. The young boy suffering from the same congenital disorder remained seizure free for two months. The girl suffering from temporal lobe epilepsy showed atypical EEG readings during the initial evaluation and still had severe seizures post-operatively. No patient exhibited new post-operative neurological deficits.

For all the cases, the 3D imaging allowed the accurate evaluation of the lesions' anatomic location and relationship with neighboring cortical functionally relevant areas. The following cases illustrate the applications of our system.

Case 1: A seven year old boy with a one-year history of focal seizures and a lesion in the parasagittal posterior frontal lobe which on the MRI seemed consistent with hamartoma or dysplasia was admitted for subdural grid and strip placement and long-term monitoring. During the initial operation, 96 electrodes were applied directly to the cortical surface, including one posterior interhemispheric strip placed directly vertically down within the interhemispheric fissure posterior to the central sulcus and another placed anteriorly (Figure 2). One frontal and one parietal grid were placed subdurally on the lateral surface of the posterior frontal and the parietal lobes respectively. The location of the electrodes was recorded on the $3 \mathrm{D}$ model using the LED probe (black dots). Electrographic monitoring detected that all episodes were identical and consisted of abrupt and simultaneous onset of low amplitude fast activity in electrodes F32 
of the frontal grid and AI11 and AI12 of the anterior interhemispheric strip. Using these results and intra-operative navigation the lesion was removed with careful motor and sensory monitoring. The patient tolerated the procedure well and has remained seizure free since then.

Case 2: An 8 year old boy suffering from intractable seizures was examined. The MRI showed no apparent lesions. Previous EEG results suggested a left temporal origin for seizure which prompted the decision to insert subdural grids and strips on that area to refine the seizure focus area. The location of the grids and strips was recorded and used as a map during bedside stimulation. Cortical stimulation was performed through the indwelling grids and strip electrodes. A mild interference with language in the superior portion of the posterior portion of the left latero-temporal grid in a region corresponding to Broca's area. As a result, it was decided to conducted a left temporal lobectomy as well as hippocampectomy. The hippocampus was reconstructed, displayed on the 3D model and used for guidance during surgery (Figure 1). The patient tolerated the procedure well and remained seizure free post-operatively.

Case 3: A two and a half year old girl with a history of tuberous sclerosis and cardiac disrrhythmia was admitted for seizure focus removal using subdural grids and strips and $3 \mathrm{D}$ reconstruction and navigation. A 32-contact grid was placed over the right lateral frontal region and an 8 contact strip was placed interhemispherically on the right frontal region. During the intracranial monitoring, it was noted that the seizure onset was localized to several contacts on the grid which were in the immediate region of the cortical tuber. Using $3 \mathrm{D}$ reconstruction and navigation, the tuber which was lying directly beneath the electrodes and which had been identified as the zone of epileptogenicity was removed (Figure 3). Following the operation, the patient remained free of post-operative neurological deficits with an MRI which showed a nice resection cavity.

\section{Discussion}

The system described above provides a novel application for surgical navigation systems for epilepsy assessment and surgery. EEG investigation results are merged with MRI imaging, and 3D reconstruction, to offer a functional as well as structural map for guidance during epileptic focus resection.

For the evaluation of epilepsy cases, EEG remains the cornerstone for the localization of seizure foci [11]. Interictal as well as ictal epileptiform activity which include such abnormalities as sharp waves, spikes, focal slowing and asymmetries of beta activity are used for the localization [1]. However, it is common that the EEG results, clinical neuroimaging results and clinical findings are not congruent, in which case the procedure of subdural grid and strip evaluation become quite useful. These electrodes are used for the planning of a safe resection, especially if the source of epileptic activity is located near eloquent cortex. In such a case, functional mapping of the area surrounding the lesion using successive electrical stimulation of the electrodes is also conducted. This procedure 

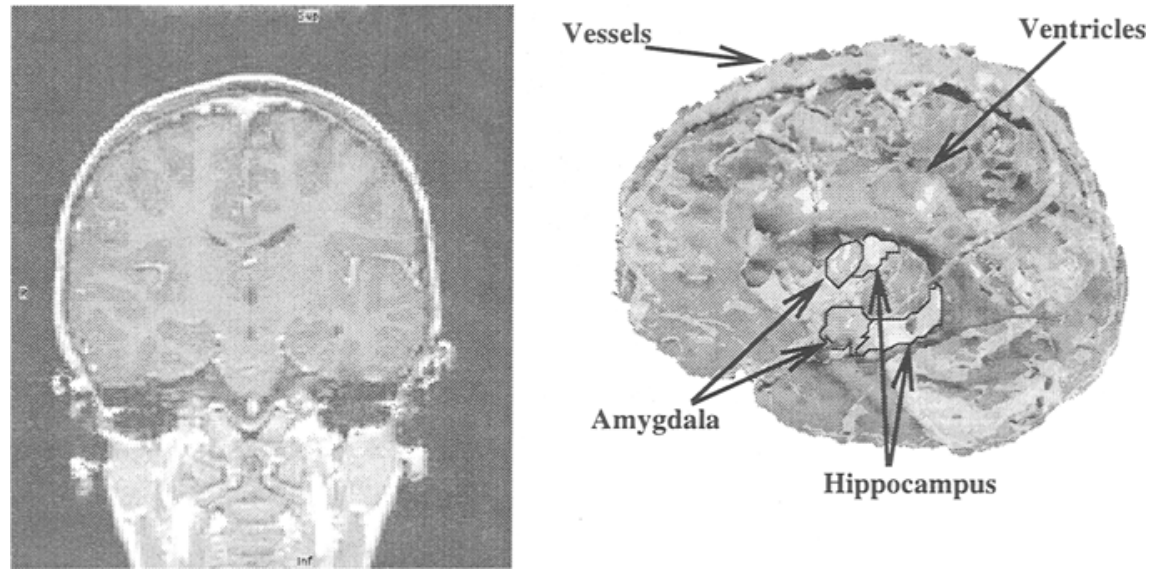

Fig. 3. LEFT: Coronal MRI showing no anatomically evident seizure foci. RIGHT: Three-dimensional reconstruction based on the patient's MRI scan used for preoperative planning and surgical guidance.

has been conducted routinely [12] and although it is associated with risks (e.g., bleeding and infection), it is tolerated remarkably well by children [13].

Because of the limitations of the surgeons' views of the brain prior to and during the surgery, they have had to create a mental picture of the lesion(s) and their spatial arrangement to neighboring structures using pre-operative EEG results and MRI scan data. By integrating the above, a physical map of the child's brain can be created, both for surgical planning and intraoperative navigation. The 3D models that are created pre-operatively in which each structure can be phased in and out, rotated in any direction and enlarged according to the view that is desired, essentially provide a means for the neurosurgical team to "look inside the brain". Moreover, the application of these models for intraoperative use, contributes to a higher definition of a lesion's location and margins. In addition, the possibility of recording the location of each electrode on the subdural grids and strips, on a model in which the brain is made more transparent and in which the lesion is labeled in a separate color, represents a great leap from the conventional two dimensional X-ray visualization.

The increased use of 3D-reconstruction [6, 15-24] and surgical navigation [2530,33-36] have substantially influenced neurosurgery. Although numerous navigation systems have been widely used for epilepsy surgery, the ability to establish a correspondence between the subdural electrodes that are placed on the brain surface and on the 3D model has not yet been described. Conventional methods of viewing these grids and strips include X-ray films [1] which in turn do not allow proper assessment of the underlying soft tissue.

We have developed the routine use of $3 \mathrm{D}$ reconstructions in surgical navigation for seizure focus removal in epilepsy surgery. Pre-operatively, the 3D model is used for surgical planning $[30,16,17]$, facilitating the evaluation of the sur- 
gical approach. Intraoperatively, this system enables recording of the subdural grid and strip electrodes directly on the 3D model, providing an intuitive way to visualize the electrodes which can easily be translated into the surgical field.

The fusion of several techniques routinely used in the operating room for neurological evaluation with imaging algorithms provides an optimal array of resources available for seizure focus evaluation and resection. The registration process, for instance, in which multiple MRI modalities including T1 and T2weighted, pre and post-gadolinium injection and $M R$ angiogram scans, can be fused together to generate the final $3 D$ model [36], substantially increases the precision of the $3 \mathrm{D}$ model and positioning of the focus with neighboring structures. The visualization of blood vessels surrounding the focus provides a useful reference point for localization. Furthermore, areas which cannot be seen on T1weighted images but which are seen on T2-weighted slices, may be combined on the same model for a more accurate map for navigation and resection.

During pediatric epilepsy surgery, the selectivity of resection is crucial, especially in foci near eloquent cortex. The ability to visualize the brain anatomy, seizure focus location and grid and strip electrodes in 3D provides an additional tool for focus localization and margin determination both pre- and intraoperatively. The fusion of modalities including EEG evaluation results, MRI scans, subdural electrode stimulation results and 3D reconstruction increases the selectivity of abnormal versus normal brain tissue and as a result, increases the likelihood of a favorable surgical outcome.

\section{Acknowledgments}

The authors thank Marianna Jacob, Mark Anderson, Caryn Sefton and Sheila Harney. This research was supported by NIH grants RO1 CA 46627-08, PO1 CA67165-01A1, PO1 AG04953-14, NSF grants BES 9631710 and DARPA grant F41624-96-2-0001. It was also supported by the Boston Neurosurgical Foundation.

\section{References}

1. Holmes GL: Intractable epilepsy in children. Epilepsia 37:14-27, 1996

2. Rosenbaum TJ, Laxer KD, Vessely M, Smith WD: Subdural electrodes for seizure focus localization. Neurosurgery 19:73-81, 1986

3. Luders H, Lesser RP, Dinner DS, Morris HH, Hahn J, Freidman L, Skipper G, Wyllie G, Friedman D: Localization of Cortical Function: New information from extraoperative monitoring of patients with epilepsy. Epilepsia 28: S56-S65, 1988.

4. Cascino GD. Structural neuroimaging in partial epilepsy. Neurosurg Clin North Am 6:455-464, 1995.

5. Kikinis R, Gleason PL, Moriarty TM, Moore MR, Alexander E III, Stieg PE, Matsumae M, Lorensen WE, Cline HE, Black PM, Jolesz FA. Computer-assisted interactive three-dimensional planning for neurosurgical procedures. Neurosurgery 38:640-651, 1996 
6. Leventon ME: A registration, tracking and visualization system for image-guided surgery. MIT Master's Thesis, May 1997

7. Gerig G, Kubler O, Kikinis R, Jolesz FA: Nonlinear anisotropic filtering of MRI data. IEEE Trans Med Imaging 11/2: 221-232, 1992

8. Cline HE, Dumoulin CL, Lorensen WE, Hart HR, Ludke S: 3D reconstruction of the brain from magnetic resonance images using a connectivity algorithm. Magn Reson Imaging 5:345-352, 1989

9. Cline HE, Lorensen WE, Kikinis R, Jolesz FA: Three-dimensional segmentation of MR images of the head using probability and connectivity. J Comput Assist Tomogr 14: 1037-1045, 1990

10. Cline HE, Lorensen WE, Souza SP, Jolesz FA, Kikinis R, Gerig G, Kennedy TE : 3D surface rendered MR images of the brain and its vasculature. Technical note $J$ Comput Assist Tomogr 14: 344-351, 1991

11. Engel J Jr: A practical guide for routine EEG studies in epilepsy: J Clin Neurophysiol 1:109-42, 1984

12. Adelson PD, Black PMcL, Madsen JR, Kramer, U, Rockoff, MA, Riviello, JJ, Helmer, SL, Mikati, M, Holmes, GL: Use of subdural grids and strip electrodes to identify a seizure focus in children. Pediatr Neurosurg 22:174-180, 1995

13. Riviello JJ Jr, Kramer U, Holmes G, . Safety of invasive electroencephalic monitoring: a comparison of adults and children. Neurology 43(suppl):A288, 1993

14. Grimson WEL, Ettinger, GJ, White SJ, Lozano-Perez T, Wells WM, Kikinis R. A automatic registration method for frameless stereotaxy, image guided surgery, and enhanced reality visualization. IEEE Trans Med Imaging 15:129-140, 1996

15. Gleason PL, Kikinis R, Altobelli D, Wells WM, Alexander E III, Black PMcL, Jolesz FA :Video registration virtual reality for nonlikage stereotactic surgery. Stereotact Funct Neurosurg 63:139-43, 1994

16. Nakajima S, Atsumi H, Kikinis R, Moriarty TM, Metcalf DC, Jolesz FA, Black PMcL: Use of cortical surface vessel registration for image-guided neurosurgery. Neurosurgery 41: 1209, 1997.

17. Nakajima S, Atsumi H, Bhalerao AH, Computer assisted surgical planning for cerebrovascular neurosurgery. Neurosurgery 41:403-409, 1997

18. Kettenbach J, Richolt JA, Hata N, et al (1997) Surgical planning Laboratory: a new challenge for radiology Computer Assisted Radiology, Elsevier, Amsterdam (in press)

19. Hu X, Tan KK, Levin DN, Galhatra S, Mullan JF, Hekmatpanah J, Spire JP: Three- dimensional magnetic resonance images of the brain: application to neurosurgical planning. $J$ Neurosurg 72: 433-440, 1990

20. Aoki S, Sasaki Y, Machida Y, Ohkubo T, Minami M, Sasaki Y: Cerebral aneurysms: detection and delineation suing 3D CT angiography. $A J N R$ 13: 11151120,1992

21. Schwartz RB, Jones KM, Chernoff DM, Mukheyi SK, Khorasani T, Tice HM, Kikinis R, Hooton SM, Stieg PE, Polak JF: Common carotid artery bifurcation: evaluation with spiral CT work in progress. Radiology 185: 513-519, 1992

22. Castillo M, Wilson JD. CT angiography of the common carotid artery bifurcation: Comparison between two techniques and conventional angiography. Neuroradiology 36: 602-604, 1994

23. Schwartz, RB. Neuroradiology applications of spinal CT. Semin Ultrasound, CT, MR 15: 139-147, 1994

24. Watanabe E, Watanabe T, Manaka S, Mayanagi Y, Takakura K. Threedimensional digitizer (neuro-navigator): New equipment of CT-guided stereotaxic surgery. Surg Neurol 27: 543-547, 1987 
25. Watanabe E, Mayanag1 $\mathrm{Y}$, Kosugi $\mathrm{Y}$, et al, Open surgery assisted by neuronavigator, a stereotactic, articulated, sensitive arm. Neurosurg 28: 792-800, 1991

26. Kato A, Yoshimine T, Hayakawa $\mathrm{T}$, et al. A frameless, armless navigational system for computer assisted neurosurgery. Technical note. J Neurosurg 74: 845- 849, 1991

27. Barnett GH, Kormos DW, Steiner CP, et al. Intraoperative localization using an armless, frameless stereotatic wand. $J$ Neurosurg 78:510-514, 1993

28. Tan KK, Grzeszczuk R, Levin DN, et al. A frameless stereotactic approach to surgical planning based on retrospective patient-image registration. Technical note. $J$ Neurosurg 79:296-303, 1993

29. Reinhardt HF, Hortsmann GA, Gratzl O. Sonic stereometry in microsurgical procedures for deep-seated brain tumors and vascular malformations. Neurosurg 32:5157, 1993

30. Barnett GH, Kormos DW, Steiner CP, et al Use of a frameless, armless, stereotactic wand for brain tumor localization and three-dimensional neuroimaging. Neurosurg 33:674-678, 1993

31. Golfinos JG, Fitzpatrick BC, Smith LR, et al. Clinical use of a frameless stereotactic arm: results of 325 cases. J Neurosurg 83:197-205, 1995

32. Friets EM, Strohbehn JW, Hatch JF, et al. A frameless stereotaxic operating microscope for neurosurgery. IEEE Trans Biomed Eng 36:608-617, 1989

33. Koivukangas J, Louhisalmi $\mathrm{Y}$, Alakuijala $\mathrm{J}$, et al. Ultrasound-controlled neuronavigator-guided brain surgery. $J$ Neurosurg 79:36-42, 1993

34. Laborde G, Gilsbach J, Harders A, et al. Computer-assisted localizer for planning of surgery and intra-operative orientation. Acta Neurochi (Wien)119:166-170, 1992

35. Robert DW, Strobehn JW, Hatch JF, et al. A frameless stereotaxic integration of computerized tomographic imaging and the operating microscope. J Neurosurg 65:545-549, 1986

36. Wells WM III, Viola P, Atsumi H, Nakajima S, Kikinis R: Multi-modal volume registration by maximization of mutual information. Medical Image Analysis 1:35$51,1996$. 